\title{
Alyssa GOLDSTEIN SEPINWALL, The Abbé Grégoire and the French Revolution. The Making of Modern Universalism
}

\section{Bernard Gainot}

\section{(2) OpenEdition}

1 Journals

\section{Édition électronique}

URL : https://journals.openedition.org/ahrf/10232

DOI : 10.4000/ahrf.10232

ISSN : 1952-403X

Éditeur :

Armand Colin, Société des études robespierristes

\section{Édition imprimée}

Date de publication : 15 mars 2006

Pagination : 211-215

ISSN : 0003-4436

\section{Référence électronique}

Bernard Gainot, «Alyssa goldstein sePInwalt, The Abbé Grégoire and the French Revolution. The Making of Modern Universalism », Annales historiques de la Révolution française [En ligne], 343 | janvier-mars 2006, mis en ligne le 17 novembre 2008, consulté le 24 avril 2022. URL : http:// journals.openedition.org/ahrf/10232 ; DOI : https://doi.org/10.4000/ahrf.10232

Ce document a été généré automatiquement le 24 avril 2022

Tous droits réservés 


\title{
Alyssa GOLDSTEIN SEPINWALL, The Abbé Grégoire and the French Revolution. The Making of Modern Universalism
}

\author{
Bernard Gainot
}

\section{RÉFÉRENCE}

Alyssa Goldstein Sepinwall, The Abbé Grégoire and the French Revolution. The Making of Modern Universalism, Berkeley, University of California Press, 2005, 307 p., ISBN

0-520-24180-0, 55 \$.

1 L'essai proposé par Alyssa Sepinwall est la synthèse de nombreux travaux menés par l'auteur sur le célèbre évêque constitutionnel de Blois. Dans le prologue, conformément à une habitude américaine qui tend à se généraliser, elle présente son parcours, qui la mena des gender studies aux réflexions sur les tensions inhérentes aux approches du genre et de la race, pour la compréhension du rapport dialectique entre l'universel et le particulier. Le thème majeur qui guide son travail est celui de "régénération », à la fois en tant qu'il est le mode sur lequel Grégoire essaye de surmonter les contradictions entre sa foi toujours affirmée de l'unité du genre humain, et les groupes spécifiques (les noirs, les juifs, les pauvres, les femmes) auxquels vont s'appliquer sa pensée et son action; et en tant que son récit s'ordonne selon les critères d'une régénération du genre biographique, qui l'amène à partir du cas individuel à explorer un champ intellectuel plus vaste.

2 De façon classique, toutefois, ce récit est construit selon une trame chronologique, qui nous permet de suivre son propos de façon très claire. Une première partie expose la rencontre entre un membre du clergé paroissial de l'Ancien Régime et les grands courants de la pensée des Lumières. Le curé d'Embermesnil y subit les influences intellectuelles diverses qui vont marquer durablement sa pensée, et s'interroge sur la condition des divers groupes sociaux, qui vont l'occuper pendant toute son existence ; 
les communautés juives ashékénazes de l'est de la France, les esclaves noirs, ses paroissiens cultivateurs. La deuxième partie couvre la décennie révolutionnaire; Grégoire s'inscrit durablement, comme législateur, et comme penseur, dans l'action concrète; la réorganisation de l'Église constitutionnnelle, l'abolition de l'esclavage, l'instruction publique. Il cherche à être le guide spirituel d'une République qui s'éloigne pourtant de ses aspirations sur bien des points. La troisième partie va du Consulat à sa mort, au début de la Monarchie de Juillet. C'est le temps des épreuves, celle d'abord de la marginalité politique, qu'il cherche à compenser par un magistère moral, et par un déplacement de l'horizon d'attente, d'une France qui ne cesse de le décevoir, vers la jeune République de Haiti, vers les États-Unis, vers les mouvements de libération nationale. Autant de malentendus et de nouvelles désillusions ; Grégoire semble de plus en plus décalé par rapport à son époque et à son image, celle de représentant intransigeant de l'héritage révolutionnaire, alors même que ses réflexions personnelles le conduisent à revenir sur les influences qui avaient été à l'origine de ses engagements. Pourtant, s'il manifeste un scepticisme croissant à l'égard de la pensée des philosophes (et prioritairement envers la croyance en la bonté originelle de l'Homme), il reste invariablement attaché au message évangélique comme unique voie du salut universel, et à la «régénération" possible de tout le genre humain par la conversion à ce message. Grégoire est jusqu'au bout l'« abbé Grégoire ».

3 L'originalité du travail d'Alyssa Sepinwall, par rapport à d'autres synthèses comme celles de Rita Hermon-Belot ou de Bernard Plongeron, est d'insister sur les contradictions inhérentes à ce programme de "régénération", ou, si l'on veut, de construction d'une république évangélique universelle. L'intérêt est tout d'abord de saisir ce parcours dans sa globalité, sans privilégier outre mesure la décennie révolutionnaire, et plus encore l'œuvre du conventionnel, pour en montrer toute la fidélité aux objectifs initiaux.

4 Avant de nous arrêter sur la partie la plus problématique, soulignons les épisodes les plus novateurs dans l'analyse d'Alyssa Sepinwall. Pour les années de formation, elle insiste sur l'influence considérable exercée sur le jeune prêtre par l'abbé Cherrier, qui fut son prédécesseur à la cure d'Embermesnil. L'abbé Cherrier insiste sur les continuités entre l'Ancien et le Nouveau Testament, pour mieux dégager le message universaliste chrétien tiré de la Genèse. Toutes les nations ont une origine commune, et ont notamment en commun l'histoire de Noë.

5 Une étude minutieuse des relations de Grégoire à cette époque, nouées lors de ses voyages dans l'est de la France, notamment à Strasbourg, amène l'auteur à relativiser l'influence janséniste, voire à nier l'exclusivité de cette influence. C'est à l'époque du Directoire que Grégoire allait s'identifier de plus en plus à l'héritage de Port-Royal, non par adhésion à une doctrine théologique, mais par identification à un mouvement religieux persécuté par l'État. Le richérisme et le gallicanisme affichés de Grégoire ne suffisent pas à en faire un janséniste. Il se sent en parfaite communion d'idées avec un mouvement plus vaste, de régénération de l'Église si on veut, qui met en avant la pureté morale, l'austérité, la simplicité primitive. Mais il subit bien d'autres influences alors : Jean-Frédéric Oberlin, un pasteur des Vosges, que Grégoire a pris pour modèle d'action paroissiale. Ou bien encore la Société des Philanthropes de Strasbourg, par l'intermédiaire de laquelle Grégoire fut sensibilisé à la question juive.

6 L'œuvre du législateur est plus connue. Alyssa Sepinwall retrace le parcours classique d'un député radicalisé par le cours de la Révolution, tant au sein des assemblées, que 
dans son évêché de Blois. Grégoire est convaincu que la régénération suppose une vigilance constante envers les ennemis de l'État; il cherche à convaincre ses fidèles que la loyauté envers le gouvernement n'est pas simple question de citoyenneté, mais un authentique commandement de Dieu. Il est particulièrement sensible à la montée du fanatisme religieux, assimilé à la contre-révolution, et il considère que les rois n'appartiennent pas au genre humain. Se pose alors l'inévitable question du régicide ; tout en estimant plausible que la lettre envoyée par les représentants en mission pour approuver la sentence de mort ait pu être signée de son nom à son insu, l'auteur laisse ouverte la question en suggérant que Grégoire ait pu justifier a posteriori l'exécution pour préserver une position rendue très délicate dans la phase de déchristianisation. Sur la question du vandalisme, Grégoire n'a pas été aussi clair, sur le moment, quant à la destruction des symboles du despotisme et de la superstition, qu'il l'a affirmé après Thermidor. Il admet alors la destruction des monuments en rapport avec le féodalisme et la tyrannie, tout en souhaitant l'édification de monuments dits « modernes ». Bref, là encore, un parcours classique de thermidorien.

7 La position de Grégoire devient plus originale sous le Directoire : foncièrement attaché au républicanisme, il l'est tout autant à la reconstruction de l'Église constitutionnelle, ce qui le place en porte-à-faux vis-à-vis de la tentative d'instauration par le Second Directoire d'une religion civile, sous le nom de théophilanthropie, qu'il condamne résolument. Sa place au sein de la classe des sciences morales et politiques de l'Institut est analysée avec finesse et minutie. L'auteur souligne son originalité au sein de la République des savants; il n'appartient à aucun des trois courants qui se partagent le magistère intellectuel de la République : les Idéologues, les partisans de la religion civile, les catholiques conservateurs. Grégoire fut l'un des promoteurs du kantisme en France, auquel il avait été initié par ses amis strasbourgeois dès 1794. Mais il allait très nettement prendre ses distances par la suite. Enfin, l'auteur accorde beaucoup d'attention aux procès-verbaux de la renaissante Société des Amis des Noirs et des colonies, rédigés par Grégoire, qui ont été récemment publiés par nos soins.

8 La troisième partie est, à mon sens, la plus neuve, sans doute parce qu'elle concerne la période la moins connue du parcours de Grégoire. Nous ne reviendrons pas sur sa marginalisation politique sous le Consulat et l'Empire, en dépit de son placard doré au Sénat, ni sur ses déboires et les constantes attaques dont il fut la cible sous la Restauration. Alyssa Sepinwall a porté son attention sur sa volumineuse correspondance, en particulier celle qui concerne les jeunes républiques de Haïti et des États-Unis, et sur certaines œuvres monumentales de cette période comme l'Histoire des sectes, ou les nombreux opuscules rangés sous l'égide De l'influence du christianisme sur tel ou tel groupe particulier (les femmes, les noirs, les indiens, les professions avilies comme les bourreaux ou les bouchers, etc.)

Grégoire était fasciné par le Nouveau Monde. Il est en correspondance avec nombre d'intellectuels à Mexico, à Bogota, au Guatemala. Il est passionné par l'épopée bolivarienne, qui affirme le droit imprescriptible des colonies américaines à l'indépendance. Mais il est surtout intéressé par les États-Unis; il correspond avec l'American Philosophical Society de Philadelphie, et avec la Société pour l'abolition de l'esclavage de Pennsylvanie. Il est toutefois très choqué des compromis passés par les dirigeants américains, dont Jefferson, sur l'esclavage. Jefferson en retour, accuse Grégoire de faire preuve de naïveté dans De la littérature des nègres, faisant remarquer que tous les noirs mentionnés dans l'ouvrage ont une portion plus ou moins importante 
de sang blanc. Grégoire, dans sa réponse, constate avec amertume que les États-Unis de 1820 sont bien plus durs dans les relations raciales que n'importe laquelle des colonies américaines. Mais c'est surtout avec son ami Joël Barlow que le malentendu est le plus évident lorsque Barlow s'en prend en 1807 dans la Colombiade à la cause conjointe des rois et des prêtres, incluant l'Église catholique dans sa charge contre la superstition.

Il éprouve les mêmes désillusions envers Haïti. Les relations avec les deux États concurrents sont minutieusement étudiées. Tandis qu'il affiche ses réserves envers le régime monarchique de Christophe, Grégoire reporte ses espérances vers le régime républicain de Pétion, sans trop prendre conscience de la nature oligarchique de ce dernier. Grégoire espérait surtout une régénération de l'ancienne colonie d'esclaves par le développement des mariages inter-raciaux, d'où devait naître une race plus robuste. La volonté du président Boyer, qui a réunifié sous son autorité le sud et le nord de l'ancienne Saint-Domingue, de passer un compromis avec le gouvernement de Charles X, met un terme à la tentative. Et c'est un Grégoire amer qui termine en 1827 son Épitre aux Haïtiens, par la formule : «Haïtiens, adieu !».

11 Que reste-t-il alors de cette période ? Un souci constant d'apologétique chrétienne, par laquelle Grégoire se rattache par bien des aspects au courant des Anti-Lumières, tout en restant fidèle à l'unité de la famille humaine. Ce qu'il reproche à la Révolution française, c'est d'avoir trahi ce message originel pour verser dans l'impiété. Il reste donc jusqu'au bout attaché à un rêve de République chrétienne, dans laquelle il voit la clef de la "régénération ». Alyssa Sepinwall souligne tout à la fois la constance de cet engagement, et les nombreuses apories qui l'émaillent.

12 Néanmoins, sur le sens de cette "régénération ", l'analyse demande éclaircissements et approfondissements. Selon l'auteur, l'utilisation de cette notion est la clef de compréhension de la façon dont Grégoire nouait la défense de certains groupes opprimés (sont particulièrement pris en compte les noirs, les juifs et les femmes) et la libération universelle, l'intégration dans la famille universelle. D'une part, cette intégration était toujours inséparable chez Grégoire de la pastorale, d'une perspective ultime de conversion à un christianisme épuré de la corruption et de l'intolérance. Au fil du temps, cette perspective est de plus en plus teintée d'apologétique; le christianisme est l'unique message de libération universelle. En outre, l'intégration se fait toujours par le renoncement à des pans entiers de l'identité culturelle des groupes concernés ; il est particulièrement dur pour la tradition rabbinique qui enferme les juifs dans un ritualisme obscurantiste. Mais c'est également vrai pour les ancien esclaves, pour lesquels il s'agit de renoncer aux liens culturels qui les attachent encore à l'Afrique, voire de renoncer à leur être propre, et c'est tout le sens de la promotion des mariages inter-raciaux, qui doivent donner naissance à un nouveau peuple. L'auteur pense que Grégoire reste ainsi implicitement attaché à une hiérarchie des valeurs, qui préserve la position dominante des européens; c'est en ce sens qu'il entend qu'une colonisation renouvelée pourrait établir des rapports nouveaux avec l'Afrique, mais où l'apport civilisationnel se ferait à sens unique. Et, selon elle, c'est encore plus vrai pour les femmes, à propos desquelles on atteint de la façon la plus évidente les limites de l'intégration, puisque Grégoire ne s'est jamais départi à leur égard d'une approche ontologique qui en fait une catégorie particulière de la famille humaine, d'essence foncièrement différente de celle du reste de l'humanité, qui est avant tout pensée comme un monde de frères. 

l'apologie d'un Grégoire mué en icône de la libération universelle. Mais ces contradictions sont celles de son temps, et, à sa façon, l'abbé est l'un de ceux qui les a affrontées avec constance, quitte à en proposer une résolution conforme à son héritage culturel et à sa vocation de prêtre. Il aurait sans doute été intéressant de mieux faire ressortir l'originalité de la synthèse de Grégoire, en la confrontant à d'autres projets régénérateurs de son temps, comme celui de Condorcet, pour lesquels la référence chrétienne est moins prégnante. La régénération ne saurait se limiter à une table rase, selon laquelle des projets prométhéens moulent une matière informe en une nation libre ; cette version réductrice n'est certes pas celle de l'auteur. Mais la régénération est inséparable d'un " processus de civilisation » qui déstructure des groupes figés dans des identités particulières, pour fonder des individus (selon quel degré de rupture avec la communauté d'origine, c'est une autre question) qui participent à un projet porteur d'une plus grande universalité. Il semble en particulier que la notion de "perfectibilité », qui est une autre notion-clef de la réflexion philosophique de cette époque, méritait d'être rappelée, et interrogée, pour mieux comprendre à quelles sollicitations Grégoire a été soumis dans les trois étapes de son parcours, le temps de la formation, le temps du magistère, et le temps des retours sur soi.

L'étude se termine par un épilogue, très intéressant, qui retrace la postérité de la figure de Grégoire, par-delà l'anecdote des funérailles si controversées. Ce n'est pas tant la haine tenace que vouèrent au républicain évangéliste et démocrate les milieux monarchistes et l'Église établie, jusqu'à la panthéonisation récente et controversée, que nous retiendrons pour terminer que les vigoureuses contestations actuelles de son universalisme : un antisémite avoué ? Un précurseur des aventures coloniales du XIX siècle, vigoureusement nourri de la conviction que la race blanche a une mission vis-àvis des races inférieures? Un misogyne impénitent et hypocrite? Et même l'initiateur d'un véritable " génocide culturel » des cultures minoritaires de l'hexagone, tel que le présente en 1989 le maire de Marseille dans une excellente recension des opinions contradictoires sur le personnage que l'auteur a l'heureuse initiative de placer en exergue de son ouvrage? Le livre d'Alyssa Sepinwall a le grand mérite de nourrir avec subtilité et nuances un débat qui place l'évêque de Blois au cœur des passions actuelles. Grégoire ne fait toujours pas consensus, et c'est une bonne chose. 